\title{
Emerging technologies in artificial ocular devices: A systematic review
}

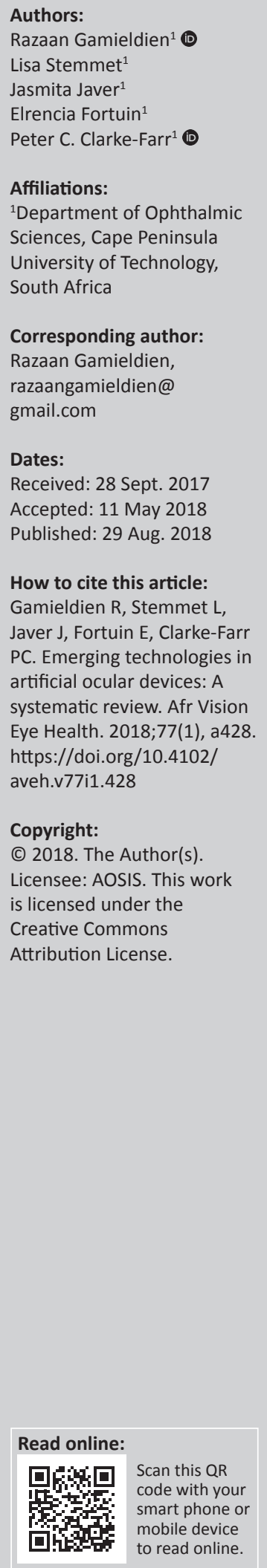

\section{Authors:}

Lisa Stemmet

Affiliations:

Department of Ophthalmic

University of Technology,

South Africa

Corresponding author:

Dates:

Accepted: 11 May 2018

artificial ocular devices: A

ematic review. Afr Vision

https://doi.org/10.4102/

Copyright:

(C) 2018. The Author(s).

Licensee: AOSIS. This work

mobile device

to read online.
Background: Recent developments in vision restoration include visual prostheses designed to electrically stimulate artificial vision in those who have lost their sight. Major efforts in this area include multi-electrode arrays surgically implanted at various placement areas throughout the visual pathway. Visual prosthetic devices are named according to these placement areas (cortical, optic nerve, sub-retinal and epiretinal). These devices attempt to restore sight for retinal degenerative diseases such as Retinitis Pigmentosa (RP) and Age-related Macular Degeneration (AMD).

Aim: To summarise the emerging technologies in the development of artificial ocular devices.

Methods: The search methodology comprised seven databases for articles published between January 2000 and March 2017. Outcome data were analysed descriptively with results summarised in a Microsoft Excel database. Both quantitative and qualitative methodologies were used, and the main findings are discussed in a narrative format.

Results: Eighty-nine full-text articles were included in this systematic review.

Conclusion: The primary goal of these artificial devices is to provide functional vision in order to perform normal daily activities. Even though recent clinical trials in certain countries have shown advances in the development of various vision-restoring devices, they do not produce the same experience for the majority of patients and are unable to completely restore normal vision. The most common type of device according to its placement is the epiretinal device which is also the most successful device as determined in the majority of clinical trials.

\section{Introduction}

According to the World Health Organization (WHO), more than 161 million individuals are visually impaired; of these, 124 million have low vision and 37 million of them are blind worldwide. ${ }^{1}$ Visual impairment consists of low vision and blindness. ${ }^{2}$ Low vision is defined as visual acuity less than $6 / 18$ but equal to or better than $3 / 60$ in the better eye with the best possible correction, and blindness is defined as visual acuity less than 3/60 or a corresponding visual field of less than 10 degrees from the point of fixation in the better eye with the best possible correction. ${ }^{2}$ Visual impairment and the number of blind-patient cases are increasing owing to age-related causes as well as chronic diseases such as uncontrolled diabetes. ${ }^{1}$ Thus, blindness related to ageing and both ocular and systemic disease will continue to increase unless major efforts and innovative technologies are developed in order to overcome this issue.

In the normal visual pathway, light transmitted from an object travels through the transparent media of the eye to eventually form an image on the light-sensitive area known as the retina. ${ }^{3}$ Sight becomes impaired when this sensitive area is damaged owing to ocular injury or disease which may eventually result in the loss of functional vision. Retinitis Pigmentosa (RP) and Age-related Macular Degeneration (AMD) are retinal diseases that affect the photoreceptors of the retina. ${ }^{4}$ Photoreceptors are specialised cells that convert light into electrical impulses which are later understood as images in the brain. Approximately, 1.5 million individuals are affected with RP, making it the leading cause of inherited blindness. ${ }^{5}$ There is no effective cure for RP or AMD, making low vision rehabilitation invaluable to affected individuals. ${ }^{6}$ Since 1755 , efforts have been made to electrically stimulate artificial vision. ${ }^{7}$ In recent years, various research groups have begun successfully implanting artificial prostheses to aid individuals with visual loss. Artificial retinal prostheses are designed to produce visual perception in order to remove the limitations that prevent affected individuals from performing normal daily tasks such as reading, the ability to recognise faces and to negotiate unfamiliar spaces. ${ }^{8}$ However, artificial visual prostheses cannot replace the complexity of the mammalian visual pathway. Their goal is not to produce vision in all of its details but to rather 
provide visual perception useful to perform daily tasks. ${ }^{8}$ This approach to aid visual loss may offer hope to individuals in that electrical stimuli might be able to bypass damaged neural tissue of the visual pathway and generate visual perception where none would otherwise exist. ${ }^{8}$

Advances in artificial hearing devices such as the Cochlear implant (auditory) gave rise to pioneering research in the field of visual implants. ${ }^{9}$ Visual prostheses were an approach to the treatment of blindness which is a relatively new area in ophthalmic sciences and ophthalmology. ${ }^{10}$ Owing to the very recent advances in the technology and intellectual property driving this area of research, there appears to be only a very limited amount of collated literature available relevant to artificial retinal and vision restoration prostheses. The restoration of sight to the blind is the most daunting challenge in ophthalmic research. ${ }^{10}$ As the numbers of blind cases are constantly escalating, the field of visual prostheses grew from a patient's dream to early patient trials..$^{10}$ It is only with continuous research efforts from teams worldwide that these individuals may be able to regain their sight. ${ }^{10}$ There is a significant demand to better understand perceptual and physiological aspects of visual prostheses with increasing advances in research and clinical tests. ${ }^{11}$ Owing to the limited amount of information pertaining to this area of ophthalmic sciences and optometry, the authors considered it important to conduct a systematic literature review in order to elicit and evaluate all the information currently available. The overall aim of this study was therefore to perform a systematic literature review by selecting and systematically appraising peer-reviewed literature on the advancement of artificial ocular devices to elucidate the most successful emerging technologies.

Four objectives were developed in order to achieve the aim of this study:

Objective 1: To perform a literature review pertaining to artificial ocular devices for the period from January 2000 to March 2017.

Objective 2: To determine the pathological conditions for which these artificial devices were developed.

Objective 3: To assess the systems used to develop artificial vision restoration.

Objective 4: To identify the most successful emerging technologies for artificial ocular devices.

\section{Methods \\ Research design}

A systematic review including both quantitative and qualitative methodologies was employed for this research.

\section{Sources of information and search strategy}

Information for this study was extracted from peer-reviewed journals located within academic databases. The search strategy involved specific keywords, timelines and language, utilised to narrow down best-suited journal articles that could be used to extract information for this study. The search for papers was conducted in two phases.

\section{Phase one}

We performed a thorough search of relevant articles from scientific databases between January 2000 and March 2017. The first phase utilised the following data bases: Cochrane Library, EBSCOhost, Google Scholar, Medline, PubMed, Wiley Online Library, Science Direct and Second Sight, using specific search criteria. Peer-reviewed journal articles, full-text and specific publication dates were included in the search strategy. All abstracts were summarised and thereafter, abstracts that met the inclusion criteria were used to obtain the full articles.

\section{Phase two}

Once a completed list of peer-reviewed full journal articles had been selected, two reviewers were chosen separately to review the journal articles and relevant data and thereafter determine if they had met the requirements and criteria for inclusion in this study. Once the final list of journal articles was compiled, a third reviewer then confirmed the selection of articles to be included. Once the data had been extracted, a critical appraisal was undertaken to systematically analyse the information according to the study designs. Article data were extracted and captured onto a Microsoft Excel database. Extracted information from included papers were summarised in categories according to year of publication, author, title, source and type of publication, language, keywords, name of featured device, area of implantation, features and functions, ocular conditions for intended treatment, components/tools needed for operation, optics involved in operation, advantages and disadvantages, clinical trial information (procedures, results, patient criteria), study design and references.

\section{Inclusion criteria}

The criteria for inclusion were as follows:

- Language: The search was conducted for English full-text or English abstracts only.

- Keywords: The following specific keywords were used to search for the included articles in peer-reviewed journals: artificial retinal devices, bionic eye, retinal implants, visual implants and visual prosthesis.

- Type of journals: Peer-reviewed scientific journals.

- Dates: From January 2000 to March 2017.

- Content: Articles that contained content of systems and technologies used in the operation of the devices (even if it contained animal studies, the technologies used were included).

\section{Exclusion criteria}

The criteria for exclusion were as follows:

- Abstracts that were not in the English language, such as those in Chinese, German and Japanese. 
- Non-peer-reviewed scientific journal articles.

- Articles published prior to January 2000.

- Articles that contained animal studies with no mention of technologies used for the devices.

\section{Results \\ Study selection}

A total of 130 articles were identified. After a preliminary review, 15 abstracts were removed as they were duplicates. Of the 115 full-text articles, a further 26 were excluded owing to lack of evidence of peer review, keywords that did not match the inclusion criteria or papers that were published outside of the specified research period. A total of 89 articles remained and were eligible for data extraction and analysis. This formed the total number of articles included for the systematic review.

\section{Study characteristics}

Table 1 provides a summary of the main characteristics of the included papers. The search strategy found articles published between January 2000 and March 2017. Most of the articles $(n=89)$ that were included were from January 2012 to March 2017 which suggests that the research surrounding retinal prostheses is growing. According to Table 1, the United States of America (USA) $(n=32)$ and Australia $(n=28)$ were the forerunners in the field of artificial visual prostheses. Types of publications included case reports $(n=3)$, clinical $(n=15)$ or experimental studies $(n=15)$, news reports published in academic journals $(n=21)$, reviews $(n=11)$, simulation $(n=21)$ or surgical studies $(n=3)$. Devices commonly reviewed and identified were the Epiret3 $(n=13)$, Alpha- IMS $(n=20)$ and the Argus ${ }^{\circledR}$ II Retinal Prosthetic System $(n=48)$. Amongst these retinal devices, some articles also discussed cortical devices and bionic eyes $(n=34)$. Various research groups have been identified as well as manufacturers including Second Sight Inc.

Articles identified pathological conditions owing to injury or disease that affected the cells of the retina. The conditions that were addressed in the articles more recently were $\mathrm{RP}(n=29), \mathrm{AMD}(n=3)$ and both (RP and AMD) $(n=38)$. Functions of devices reviewed included the restoration of visual perception, to improve visual acuity and to offer functional vision to affected individuals. Components necessary for the device to function differed depending on the type of the device such as according to the area of placement during surgical procedures. Common components identified were spectacles, a Video Processing Unit (VPU), a camera and a multi-electrode array. Articles that were intended to report on clinical trials focused on the patient criteria, the outcomes and results. Patient criteria included were visual impairment (with or without RP or AMD) or patients that were blind. In some articles, the particular age for patient criteria was 50 years or older and 18 years or older. Patients included in clinical trials needed to have some form of useful vision or light perception available. Another requirement was specific visual acuities such as less than $2.9 \log$ MAR.

Advantages and disadvantages were difficult to identify as not all articles focused on specific devices but rather on artificial prostheses in general. Advantages and disadvantages of the devices were based on the quality of vision gained from the device as well as the possible adverse effects of surgical implantation and electrical stimulus (stimulating the area of interest with electrodes).

\section{Discussion}

Research attempting to restore functional vision in individuals affected with RP and AMD has been ongoing for more than 30 years, $^{12}$ but artificial retinal prostheses have achieved major goals in recent years. Information about the visual world is converted into electrical signals to restore lost vision. ${ }^{13}$ This technique of electronic restoration is viewed as a safe and viable option for individuals who have lost substantial numbers of photoreceptors and who suffer from severe visual impairment according to these authors. ${ }^{13}$ Recent studies have shown that for some patients, restoration to the extent of reading large letters $(8.5 \mathrm{~cm}$ high, $1.7 \mathrm{~cm}$ line width, corresponding to a height of approximately 9 degrees of visual angle at $60 \mathrm{~cm}$ testing distance) is possible. ${ }^{14}$ Visual acuity and natural light perception have continually improved by introducing an increase in the amount and density of electrodes used to produce light spots known as phosphenes. However, an increase in the number of electrodes results in larger devices that need to be produced. Larger devices require more energy and thus generate more

\begin{tabular}{|c|c|c|c|c|c|c|c|c|c|}
\hline Type of paper & $n$ & Country of origin & $n$ & $\begin{array}{l}\text { Type of device } \\
\text { (according to placement) }\end{array}$ & $n$ & Year of publication & $n$ & $\begin{array}{l}\text { Year of publication } \\
\text { (cont.) }\end{array}$ & $n$ \\
\hline Case reports & 3 & USA & 32 & Epiretinal & 28 & From Jan 2000 & 3 & 2009 & 5 \\
\hline Experimental studies & 15 & Europe: Germany, Netherlands, etc. & 10 & Transchoroidal & 1 & 2002 & 4 & 2011 & 4 \\
\hline News reports & 21 & New Zealand & 1 & Suprachoroidal & 1 & 2003 & 0 & 2012 & 15 \\
\hline Reviews & 11 & China & 9 & Visual prosthesis (general) & 21 & 2004 & 2 & 2013 & 10 \\
\hline \multirow[t]{3}{*}{ Surgical studies } & 3 & India & 2 & Visual Cortex & 10 & 2006 & 0 & 2015 & 13 \\
\hline & - & Australia & 28 & - & - & 2007 & 0 & 2016 & 4 \\
\hline & - & - & - & - & - & 2008 & 3 & Till March 2017 & 1 \\
\hline Total & 89 & & 89 & & 89 & & - & & 89 \\
\hline
\end{tabular}

USA, United Stated of America; UK, United Kingdom.

$n$, number. 
heat, which may lead to complications during surgical implantation. ${ }^{4}$ However, epiretinal devices, such as the Argus II, require less invasive surgery. Furthermore, various engineering and biological factors may need to be considered to improve the visual performance of the prosthetic devices. Another treatment for retinal degeneration, gene therapy has proved to be safe and effective for those who suffer from Leber's congenital amaurosis which is the most severe form of inherited retinal degeneration. ${ }^{13}$ However, RP can be inherited in an autosomal dominant fashion which makes current gene therapy less viable and effective. ${ }^{13}$ Therefore, the technology field of retinal prosthetic devices is amongst the most promising interventions to aid such individuals suffering from late stage retinal degeneration. ${ }^{13}$

\section{Types of vision restoration devices}

A review of the included articles demonstrates that artificial retinal devices are categorised according to the placement of electrodes during the surgical procedure. The three broad categories of devices evident in the reviews are outlined below. The optic nerve and lateral geniculate nucleus provide an alternative stimulation target discussed below. ${ }^{15}$

\section{Visual cortex stimulation}

Blindness caused by glaucoma, diabetic retinopathy and trauma may result in a non-functional retina. ${ }^{16}$ In these cases, there are limited therapeutic options. As a retinal prosthesis is unable to bypass the damaged retina to produce useful vision, electrical stimulation at the visual cortex would be ideal. ${ }^{16}$ The idea to electrically stimulate vision at the visual cortex began in the 1930s where neurologists demonstrated that phosphenes were evoked using localised electrical stimulation of special regions of the cerebral cortex. ${ }^{16}$ Brindley and his colleagues pioneered the first clinical trial which included an 80-electrode array placed over the visual cortex. ${ }^{16}$ Trial patients perceived reproducible phosphenes, but this device was limited owing to the technological resources available at that time. ${ }^{16}$ With diseases causing optic atrophy the visual cortex also needs to be stimulated. ${ }^{17}$ Clinical trials in the 1970s and 1980s demonstrated functional visual recovery with a 100-electrode array placed on the visual cortex. ${ }^{17}$ However, the lack of constant functional vision brought the clinical trials to an end. ${ }^{17}$ There are several research groups experimenting with cortical devices that are equipped with several hundred electrode arrays. ${ }^{15}$

\section{Optic nerve stimulation}

Optic nerve prosthesis may be surgically implanted in patients with surviving retinal ganglion cells and an intact optic nerve. ${ }^{15}$ Trials performed concluded that recipients were able to recognise complex shapes, and perform object localisation, discrimination and grasping. ${ }^{15}$ Currently, research groups are still in the process of developing devices that utilise electrodes that may stimulate the optic nerve or the optic disc. ${ }^{15}$

\section{Lateral geniculate nucleus stimulation}

This stimulation target is considered favourably owing to the lateral geniculate nucleus (LGN) having compact dimensions, retinoptic organisation and motor and colour pathways being physically separated. ${ }^{15}$ Stimulating the LGN for functional vision could generate visual cortex responses very similar to those elicited by natural vision. ${ }^{15}$ There is still much work to be done in this area of artificial ocular prosthesis as research groups are aiming to develop a device which can provide functional vision. ${ }^{15}$

\section{Sub-retinal devices}

These devices are implanted in the common location where photoreceptors are found in the retina. ${ }^{4}$ During surgery, the electrode array is most likely to be placed between the bipolar cell layer and the retinal pigment epithelium. ${ }^{18}$ Sub-retinal prostheses can be divided into passive and active systems. ${ }^{4}$ The first passive sub-retinal device was developed by Optobionics Corporation and was known as the Artificial Silicon Retina; this device uses incident light to produce electrical stimulation. ${ }^{18}$ However, incident light contains only a limited amount of energy to be converted into an electrical stimulation and was therefore insufficient for the conversion of electrical stimulation into phosphenes and hence unable to elicit functional visual perception. Visual outcomes summarised from an 18-month study with retinal degeneration that included ten patients showed subjective improvement in visual function in six patients. ${ }^{18}$ Currently, there are no passive systems being utilised. ${ }^{4}$ In active systems, the incident luminance is converted into a graded electrical current by the use of amplifiers in each pixel, thereby creating an electronic image, which is then transmitted pixel-by-pixel to the bipolar cells of the retina. This electronic image is then perceived as shades of grey.

With sub-retinal systems, no light adaptation similar to that of a normal visual pathway is possible. ${ }^{4}$ Even so, one of the main advantages of the sub-retinal prostheses is that light perception feels natural because the remaining parts of the visual pathway are used and information that is being processed in the inner retina may be maintained and used to produce natural light perception. Natural eye movement is essential for natural light perception; hence, another advantage of the sub-retinal device is that the placement of the electrode array implanted inside retina of the eye allows for natural eye movements. ${ }^{4}$ Sub-retinal devices allow for higher resolution images and forms and shapes but because implantation surgery is highly complicated with this type of device, epiretinal prosthetic devices are likely to be more successful in terms of implantation and producing functional vision. The Alpha-IMS developed by Retina Implant AG (Reutlingen, Germany) is the only sub-retinal device that has been applied in patients for clinical trials. ${ }^{4}$ Unlike epiretinal devices, the Alpha-IMS does not depend on an external camera. Instead, it uses a multi-photodiode array. According to two recent studies that included eleven and three patients, respectively, eight of the eleven patients reported visual 
percepts with a $\log$ MAR acuity of $1.78(6 / 360) \cdot{ }^{13}$ In the other study, all three patients had light perception; two of these patients had spatial resolution of 0.46 cycles $/ \mathrm{deg}^{-1}$, while one patient read letters that were $8.5 \mathrm{~cm}$ high $($ at $60 \mathrm{~cm}$ ) and all three patients were able to localise objects on a table. ${ }^{14}$

\section{Epiretinal devices}

These devices are implanted on top of the ganglion cell layer (within nerve fibre layer of the retina). The electrode array is attached to the retina via retinal tacks during surgical implantation. ${ }^{4}$ Hence, ganglion cells are directly stimulated. Image information is transmitted via an external camera mounted on spectacles where an external camera captures the image and allows for magnification and zoom of the target being viewed. ${ }^{4}$ This enables functional artificial vision to be optimised despite a low number of pixels in the electrode array. The advantages of the device include the wearable portion of the device which permits easy upgrades without requiring subsequent surgery, and this electronic technology allows the patient and doctor to gain full control over every electrode section and digital signal processing involved in the imaging of objects; thus, the device can be personalised/customised for individual patients. ${ }^{10}$ The disadvantages of this device include the need for technology to provide a prolonged adhesion of the device to the inner part of the retina, and increased electrical current is necessary owing to the greater distance between the bipolar cells and the epiretinal cells (compared to that of a sub-retinal device). ${ }^{10}$ Another disadvantage of the external camera is that it eradicates natural eye movements which impacts natural visual perception. ${ }^{18}$ To date, the Argus II, EPI-RET 3 and Intelligent Medical Implants (IMIs) have been developed and clinical trials have been performed with these devices.

The Argus II is the first epiretinal prosthesis approved in clinical trials in the USA and Europe. This device comprises a 60-electrode array. ${ }^{12}$ This device, also known as the 'bionic eye' $^{\prime}$, is intended to electrically stimulate the retina to induce visual sensation in individuals who have lost their vision. ${ }^{19}$ This device is indicated to aid those individuals with severe to profound RP and the device comprises external and internal components to provide its function. ${ }^{19}$ This system is implanted in one eye only (typically the worst eye) of blind patients. ${ }^{20} \mathrm{~A}$ miniature video camera is situated in the patient's spectacles which then captures a scene and this video is then transferred to a small patient-worn computer known as the VPU. ${ }^{19}$ The VPU processes information into instructions that are then sent back to the spectacles via a cable. ${ }^{19}$ The VPU can be worn as a shoulder strap as well as a belt. ${ }^{20}$ At this point, these instructions are wirelessly transmitted to an antenna in the retinal implant that has been surgically placed in the retina. These signals are then sent to a multi-electrode array which emits tiny impulses of electricity to bypass the damaged photoreceptor cells and stimulate the remaining cells of the retina. ${ }^{19}$ The Argus II is implanted in the worst eye and does not promise to restore natural/normal functional vision once experienced. ${ }^{19}$ It is not intended to slow or reverse the progression of RP and will not replace normal low vision aids, but it should allow for some functional vision to be attained. ${ }^{19}$

Spatial resolution appears to be the main limitation in artificial retinal devices which could be optimised by increasing electrical stimulation. This, however, gives rise to various safety concerns. ${ }^{12}$ The EPI-RET 3 and Intelligent Medical Implants also utilise an external camera and both are presently in clinical trials in Europe. ${ }^{4}$ The Argus II is currently the only device that has received FDA (Food and Drug Administration) approval. ${ }^{2}$ Both the Argus II as well as the Alpha-IMS has received the European CE Marking. ${ }^{4}$ Other devices are currently in human clinical trials except the Boston Retinal Implant which is undergoing animal studies. ${ }^{4}$ In terms of resolution, visual resolution theoretically collates with the amount of electrodes present on a multi-electrode array. ${ }^{4}$ The Boston Retinal Implant leads with 100 electrodes, the Argus II with 60 electrodes and a visual acuity (VA) of 20/1262, whereas the Alpha-IMS delivers a VA of 20/546. ${ }^{4}$ Although the Argus II is leading in terms of successful patient cases and implantation, the Alpha-IMS is most likely to achieve long-term success in the future beyond the current clinical availability of the device. ${ }^{4}$

\section{Clinical trials}

Visual prostheses have evolved to some degree for restoring basic visual function in blind individuals taking part in current clinical trials. ${ }^{21}$ Retinal prostheses are the most advanced form of visual prostheses and account for the greatest amount of completed and ongoing clinical trials. ${ }^{13}$ A sub-retinal approach, the active micro photodiode array (MPDA) has been implanted in most human subjects to date compared to other sub-retinal devices. ${ }^{21}$ Patients were also clinically followed up for the longest period after the device had been implanted; however, epiretinal prostheses have a longer history of successful implantation. ${ }^{21}$ Intelligent Medical products (IMI) $\mathrm{GmbH}$ performed successful implants in four subjects with a 49-electrode array for 18 months, while the Epi-Ret was implanted in six subjects with a 25-electrode array for one month. ${ }^{21}$ The Argus I and II retinal systems continue to be the only long-term trials to date, while the Argus II epiretinal prosthesis has been surgically implanted into numerous subjects with profound RP as part of a worldwide clinical feasibility study where approximately one-third of subjects experienced measurable improvements in visual acuity. ${ }^{21}$ In November 2014, a legally blind female patient, who became blind at the age of 42 owing to RP, became the first person in the USA to receive the Argus II bionic device. ${ }^{22}$ Approximately one-third of the subjects experienced measurable improvements in visual acuity. ${ }^{21}$ The Alpha-IMS has been tested in nine subjects to date and has received regulatory approval in Europe, ${ }^{23}$ while in 2013 the Bionic eye (Argus II) was approved in the USA and Europe. Bionic Vision Australia completed a clinical trial of a suprachoroidal implant with three patients showing sustained perception, stability of implantation and also significantly 
improved light location detection over a 12-month period. ${ }^{24}$ So far, all reported outcomes to date have been in patients with end-stage RP and choroideremia. ${ }^{24}$ The Argus II has been made available in 2014; however, these devices are not available or approved in South Africa as yet. ${ }^{25}$

\section{Suggested model for retinal devices}

Based on the review of the scientific papers, the authors have identified certain aspects required for successful restoration of visual function. Ideally, the artificial retinal device should be implanted on top of the ganglion cell layer of the retina (as with epiretinal devices) to directly stimulate the ganglion cells and bypass the other retinal layers which may be damaged owing to pathology. This means that the model will provide visual perception to individuals affected with RP or AMD even if the other retinal layers have been damaged. This model should consist of a 600-electrode array to elicit a greater amount of phosphenes, thus enabling patients to perform a wider variety of tasks. In order for a 600-electrode array to be developed, the implications of stimulating vision through the use of electricity must first be overcome as the greater number of electrodes implanted will require a greater amount of electricity which potentially may do more harm than desired.

\section{Conclusions and recommendations}

One of the most feared and debilitating physical disabilities is that of blindness. ${ }^{26}$ Blindness as a result of pathological diseases such as RP or AMD are incurable and irreversible which leads to a drastic reduction in the quality of life of affected individuals. ${ }^{27}$ These individuals may require more medical attention and assistance to perform their daily activities. According to an estimation made by the AMD International Alliance, blindness and visual impairment has added cost to the world's economy of nearly 2.3 trillion Euros in 2010. ${ }^{27}$ This estimation is based on the medical expenses of 733 million Euros for blind or severely visually impaired individuals worldwide and also the value of time caring for them and the loss of productivity, which result in loss of tax revenues that ultimately aid the health care systems in place. ${ }^{27}$ If the implantation of a visual prosthetic device aids affected individuals in carrying out daily tasks and can provide support to basic skills that can aid them in gaining an income to sustain their quality of life, then the cost justifies the expense. After a review of available literature, it appears that there are two devices commercially available for the successful treatment of visual impairment: the Argus II Retinal Prosthesis System manufactured by Second Sight Medical Products (California, USA) and the Alpha-IMS device manufactured by Retinal Implant in Reutlingen, Germany. Based on this systematic review, prospects are good for successful restoration of visual function, and emerging technologies are expected to develop exponentially. Options such as optic nerve and visual cortex stimulation may become viable options in the future if limitations such as surgical risks of infection and intracranial haemorrhaging may be overcome. ${ }^{7}$ Cortical prosthesis that may bypass the retina and be directly stimulated will allow for higher resolution images/pixels owing to the more tightly packed electrodes. ${ }^{7}$ The benefits of such ocular devices will go a long way to alleviate the functional, economic and psychological impact that vision loss has on affected individuals.

\section{Acknowledgements Competing interests}

The authors declare that they have no financial or personal relationships that may have inappropriately influenced them in writing this article.

\section{Authors' contributions}

R.G. was the leader and primary contributor to the writing of this article with assistance from L.S., J.J. and E.F. were involved in the data collection and preparation of the group report. P.C.C-F. supervised the student project, reviewed the article and provided input and guidance on the writing, structure and article content.

\section{References}

1. Resnikoff S, Pascolini D, Etya'ale $D$, et al. Global data on visual impairment in the year 2002. Bull World Health Organ [serial online]. 2004;82(11):844-851. Retrieved January 28, 2015, from: https://www.researchgate.net/publication/8090259 Global_data_on_visual_impairment_2002

2. World Health Organization. Catalogue of key eye health indicators in the African Region. Geneva World Health Organ [serial online]. 2017. Retrieved 02 May, 2018, from: http://apps.who.int/iris/bitstream/handle/10665/254834/978929023289eng. pdf?sequence $=1$ \&isAllowed $=y$

3. Tunnacliffe AH. Introduction to visual optics. 4th ed. London: Association of British Dispensing Opticians; 1993.

4. Lin TC, Chang HM, Hsu CC, et al. Retinal prostheses in degenerative retinal diseases. J Chin Med Assoc. 2015;78:501-505. https://doi.org/10.1016/j.jcma. 2015.05.010

5. Humayun MS. Intraocular retinal prosthesis. Trans Am Ophthalmol Soc [serial online]. 2001 [cited 2018 Apr 26];99:271-300. Available from: http://www.ncbi. nlm.nih.gov/pubmed/11797315

6. Vukicevic M, Le A, Baglin J. Research report: A simplified method of identifying the trained retinal locus for training in eccentric viewing. Journal of Visual Impairment and Blindness, 2012; p. 555-562.

7. Greenberg RJ. Visual prostheses: A review. Neuromodulation. 2000;3:161-165. https://doi.org/10.1046/j.1525-1403.2000.00161.x

8. Maynard EM. Visual prostheses. Annu Rev Biomed Eng [serial online]. 2001;3: 145-168. Retrieved September, 2015, from: http://www.annualreviews.org/doi/ abs/10.1146/annurev.bioeng.3.1.145\nhttp://www.ncbi.nlm.nih.gov/pubmed/ 11447060

9. Xia Y, Peng X, Ren Q. Retinitis pigmentosa patients' attitudes toward participation in retinal prosthesis trials. Contemp Clin Trials. 2012;33(4):628-632. https://doi. org/10.1016/j.cct.2012.03.007

10. Banarji A, Gurunadh VS, Patyal S, Ahluwalia TS, Vats DP, Bhadauria M. Visual prosthesis: Artificial vision. Med J Armed Forces India. 2009;65:348-352. https:// doi.org/10.1016/S0377-1237(09)80098-1

11. Chen SC, Suaning GJ, Morley JW, Lovell NH. Simulating prosthetic vision: I. Visual models of phosphenes. Vision Res. 2009;49:1493-1506. https://doi.org/10.1016/j. visres.2009.02.003

12. Dowling J. Current and future prospects for optoelectronic retinal prostheses. Eye. 2009;23:1999-2005. https://doi.org/10.1038/eye.2008.385

13. O'Brien EE, Greferath U, Vessey KA, Jobling Al, Fletcher EL. Electronic restoration of vision in those with photoreceptor degenerations. Clin Exp Optom. 2012;95: 473-483. https://doi.org/10.1111/j.1444-0938.2012.00783.x

14. Zrenner E, Bartz-Schmidt KU, Benav H, et al. Subretinal electronic chips allow blind patients to read letters and combine them to words. Proc R Soc B Biol Sci [serial online]. 2011;278(1711):1489-1497. Retrieved May 07, 2018, from: http://www. ncbi.nlm.nih.gov/pubmed/21047851

15. Lewis PM, Ackland HM, Lowery AJ, Rosenfeld JV. Restoration of vision in blind individuals using bionic devices: A review with a focus on cortical visual prostheses. Brain Res. 2015;1595:51-73. https://doi.org/10.1016/j.brainres.2014.11.020

16. Shepherd RK, Shivdasani MN, Nayagam DAX, Williams CE, Blamey PJ. Visual prostheses for the blind. Trends Biotechnol. 2013;31: 562-571. https://doi.org/ 10.1016/j.tibtech.2013.07.001

17. Picaud S. Retinal prostheses: Clinical results and future challenges. CR Biol. 2014;337(3):214-222. https://doi.org/10.1016/j.crvi.2014.01.001 
18. Ong JM, da Cruz L. The bionic eye: A review. Clin Exp Ophthalmol. 2012;40:6-17. https://doi.org/10.1111/j.1442-9071.2011.02590.x

19. Second Sight. Argus ${ }^{\circledR}$ II Retinal Prosthesis System [homepage on the Internet]. Retrieved May 02, 2018, from: http://www.secondsight.com/argus-ii-retinalprosthesis-system-en.html

20. Ho AC, Humayun MS, Dorn JD, et al. Long-Term results from an epiretinal prosthesis to restore sight to the blind. Ophthalmology. 2015;122(8):1547-1554. https://doi.org/10.1016/j.ophtha.2015.04.032

21. Ahuja AK, Behrend MR. The Argus ${ }^{\mathrm{TM}}$ II retinal prosthesis: Factors affecting patient selection for implantation. Progr Retin Eye Res. 2013;36:1-23. https://doi. org/10.1016/j.preteyeres.2013.01.002

22. Brady-Simmons C, Biest R Van Der, Bozeman L. Miami lighthouse for the blind and visually impaired case study: Vision rehabilitation for the first Florida resident to visually impaired case study: Vision rehabilitation for the first Florida reside
receive the Argus II 'Bionic Eye'. J Vis Impair Blind. 2016;110(3):177-182.
23. Weiland JD, Humayun MS. Retinal prosthesis. IEEE Trans Biomed Eng. 2014;61(5): 1412-1424. https://doi.org/10.1109/TBME.2014.2314733

24. Lewis PM, Ayton LN, Guymer RH, et al. Advances in implantable bionic devices for blindness: A review. ANZ J Surg. 2016;86: 654-659. https://doi.org/10.1111/ ans.13616

25. Carte Blanche. Seeing is believing television documentary. Producer: Eugene Botha, Presenter: Claire Mawisa. South Africa: M-net DSTV; 3 May 2015. Retrieved September, 2015, from: https://carteblanche.dstv.com/

26. Ayton LN, Blamey PJ, Guymer RH, et al. First-in-human trial of a novel suprachoroida retinal prosthesis. PLoS One. 2014;9(12):1-27. https://doi.org/10.1371/journal. pone.0115239

27. Vaidya A, Borgonovi E, Taylor RS, et al. The cost-effectiveness of the Argus II retinal prosthesis in Retinitis Pigmentosa patients. BMC Ophthalmol. 2014;14: $1-10$. 